Judith A.R. van Waes*, Linda M. Peelen, Hans Kemperman, Remco B. Grobben, Hendrik M. Nathoe and Wilton A. van Klei

\title{
Kinetics of troponin I in patients with myocardial injury after noncardiac surgery
}

DOI 10.1515/cclm-2016-0301

Received April 12, 2016; accepted August 29, 2016; previously published online October 12, 2016

\section{Abstract}

Background: Myocardial injury after noncardiac surgery, as measured by troponin elevation, is strongly associated with mortality. However, it is unknown in which patients prognosis can be improved. The presence of kinetic changes of troponin may be associated with a worse prognosis and warrant more aggressive management. Therefore, we aimed to study the kinetics of troponin in patients with postoperative myocardial injury, and to determine the added predictive value of kinetic changes of troponin on mortality.

Methods: This cohort study included patients with myocardial injury after noncardiac surgery. Troponin I (TnI) was measured on the first three postoperative days. The primary outcome was all-cause 1-year mortality. We studied both absolute and relative TnI changes, and determined the delta TnI that was associated with mortality to distinguish a rise-and-fall TnI pattern from a stable TnI pattern. Next, we determined the added predictive value of a rise-and-fall TnI pattern for mortality.

Results: In total, 634 patients were included. The risk ratio (RR) for mortality increased significantly with an absolute

\footnotetext{
*Corresponding author: Judith A.R. van Waes, MD, University Medical Center Utrecht, Department of Anesthesiology, Local mail: Q04.2.313, P.O. Box 85500, 3508 GA Utrecht, The Netherlands, Phone: +31 88755 5555, Fax: +31 30254 1828, E-mail: j.a.r.vanwaes@umcutrecht.nl

Linda M. Peelen: Department of Anesthesiology, University Medical Center Utrecht, Utrecht, The Netherlands; and Department of Epidemiology, Julius Center for Health Sciences and Primary Care, University Medical Center Utrecht, Utrecht, The Netherlands Hans Kemperman: Department Clinical Chemistry and Haematology, University Medical Center Utrecht, Utrecht, The Netherlands Remco B. Grobben: Department of Anesthesiology, University Medical Center Utrecht, Utrecht, The Netherlands; and Department of Cardiology, University Medical Center Utrecht, Utrecht, The Netherlands

Hendrik M. Nathoe: Department of Cardiology, University Medical Center Utrecht, Utrecht, The Netherlands

Wilton A. van Klei: Department of Anesthesiology, University Medical Center Utrecht, Utrecht, The Netherlands
}

delta TnI of $\geq 200 \mathrm{ng} / \mathrm{L}$ (RR 1.5, 99.4\% CI 1.0-2.2, $\mathrm{p}=0.003$ ). Using this delta TnI to define a rise-and-fall pattern, 459 patients $(72 \%)$ had a stable TnI pattern and 175 patients (28\%) had a rise-and-fall pattern. When added to a model including the highest TnI value and variables from the revised cardiac risk index (RCRI), the TnI pattern did not increase the predictive value for mortality.

Conclusions: A postoperative TnI rise-and-fall pattern was associated with 1-year mortality, but had no added value in addition to the highest TnI level to predict 1-year mortality. Therefore, postoperative TnI kinetics are not useful for further mortality risk stratification in patients with myocardial injury after noncardiac surgery.

Keywords: kinetics; myocardial injury; perioperative; surgery; troponin.

\section{Introduction}

Postoperative myocardial injury as measured by troponin elevation after noncardiac surgery is strongly associated with mortality [1-3]. Therefore, routine postoperative monitoring of cardiac biomarkers has been recommended to identify patients at risk [4-6].

Routine troponin I (TnI) measurements were implemented in our hospital as part of our postoperative care protocol, in order to facilitate early identification and subsequent cardiovascular optimization of patients at risk of death. However, a major problem in initiating or optimizing treatment in individual patients is the lack of knowledge of the underlying etiology of myocardial injury in the postoperative phase [7]. When the cause of the injury is often not clear, it is difficult to determine in which patients prognosis can be improved. Consequently, in more than half of the patients the clinical course is awaited without any intervention [8].

Since the introduction of high sensitivity troponin assays, the number of patients in which elevated troponin is attributable to other conditions than myocardial infarction has increased [9]. Therefore, the pattern of cardiac biomarkers over time has become an important criterion in the diagnosis of myocardial infarction. A pattern with 
a rise and/or fall of troponin above the 99th percentile of the upper reference limit in the presence of clinical signs and symptoms is considered as evidence of myocardial infarction [4]. In the non-surgical population, absolute troponin changes discriminate well between acute myocardial infarction and other causes of myocardial injury, and have high accuracy in identifying candidates for early coronary angiography [10]. Likewise, the presence of kinetic changes of troponin in the postoperative phase may reflect clinically relevant myocardial ischemia that is associated with a worse prognosis and that warrants more aggressive therapy as compared to troponin elevation without kinetic changes. This may help to identify patients who require follow up and treatment, but evidence for this hypothesis is lacking.

We therefore aimed to study the kinetics of troponin in patients with myocardial injury after noncardiac surgery, and to determine the added predictive value of kinetic changes of troponin on 1-year mortality.

\section{Materials and methods}

\section{Patients}

This observational cohort study included consecutive patients undergoing noncardiac surgery between January 1st 2011 and December 31st 2012 at the University Medical Center Utrecht, The Netherlands, a 1000 bed tertiary referral hospital. Patients were eligible if they were aged 60 years or older, were undergoing intermediate to high-risk noncardiac surgery under general or spinal anesthesia with an expected postoperative length of hospital stay of at least $24 \mathrm{~h}$. In these patients, troponin was measured routinely on the first three postoperative days as part of our postoperative care protocol. Patients who had troponin elevation after surgery were included in this study. For patients who underwent surgery more than once, the first surgery was included in the analyses. A reoperation was included as a novel case if this surgery took place at least 1 year after the first surgery. Patients were excluded if troponin was not measured or measured only once after surgery, or if patients were lost to follow-up within 1 year after surgery.

The local medical Ethics Committee waived the need for informed consent, as only routinely collected patient data were used and data were anonymized before analysis (UMC Utrecht Medical Research Ethics Committee, protocol number 11/120-C).

\section{Data collection}

All preoperative and postoperative data were obtained from electronic medical and administrative records. Data collected of all patients included patient characteristics, preoperative physical status, comorbidities including factors from the revised cardiac risk index (RCRI), [11] postoperative TnI measurements and 1-year mortality. Emergency surgery was defined as surgery required within $72 \mathrm{~h}$. High-risk surgery was defined as intrathoracic, intraabdominal or suprainguinal vascular surgery [11]. Ischemic heart disease was defined as a history of myocardial infarction or coronary revascularization. Preoperative renal insufficiency was defined as a preoperative glomerular filtration rate $<45 \mathrm{~mL} / \mathrm{min} / 1.73 \mathrm{~m}^{2}$. Chronic heart failure was defined as a left ventricular ejection fraction $<40 \%$. The municipal personal records database was consulted for mortality data.

\section{Routine postoperative troponin measurements}

According to the postoperative care protocol for older surgical patients in our hospital, the occurrence of postoperative myocardial injury was assessed routinely by $\mathrm{TnI}$ measurements. TnI was measured daily in the morning on the first three postoperative days. In case of a TnI elevation above the clinical cut-off level ( $\mathrm{TnI}>60 \mathrm{ng} / \mathrm{L})$, the ward physician was notified and a cardiac consult was requested. It was left at the discretion of the ward physician and cardiologist whether follow-up of $\mathrm{TnI}$ and further diagnostic procedures were indicated. Myocardial infarction was diagnosed by the cardiologist according to the third universal definition by judging the height of the TnI level, the presence or absence of a rise and fall in TnI, clinical symptoms, electrocardiography and/or cardiac imaging. In this, a rise and fall in TnI was not defined beforehand [4].

In the current study, for each patient all postoperative TnI measurements within 10 days after surgery were used, including follow-up measurements in addition to the routine TnI measurements. Troponin elevation was defined as a $\mathrm{TnI}>60 \mathrm{ng} / \mathrm{L}$, which was the lowest value measurable with a $10 \%$ coefficient of variation above the 99th percentile of $40 \mathrm{ng} / \mathrm{L}$ of the assay used. TnI was analyzed using the third-generation enhanced AccuTnI assay (Beckman Coulter, Brea, CA, USA).

\section{Kinetics of troponin}

For each patient we first expressed the kinetics of troponin in terms of the absolute and relative change in TnI. The absolute change in TnI was defined as the highest minus the lowest postoperative TnI value. The relative change in $\mathrm{TnI}$ was defined as the difference between the highest and the lowest postoperative TnI value, divided by the lowest TnI value.

Subsequently, we categorized patients according to their pattern of TnI changes into two categories (stable pattern and rise-andfall pattern) as follows. First, we identified potential cut-off values of the highest $\mathrm{TnI}$ value and the absolute and relative change in TnI, by grouping patients in deciles, and rounding to the nearest tenths or hundredths. For the highest TnI value, this resulted in the following TnI categories: 70-79 ng/L, 80-89 ng/L, 90-99 ng/L, 100-129 ng/L, 130-169 ng/L, 170-259 ng/L, 260-499 ng/L, 500-1499 $\mathrm{ng} / \mathrm{L}$ and $\geq 1500 \mathrm{ng} / \mathrm{L}$. Next, the risk ratio for mortality was calculated for each of these cut-off values. Because nine different cut-off values were tested, Bonferroni correction was used to adjust for multiple comparisons, resulting in a $99.4 \%$ confidence interval ( $\mathrm{p}$ value of $0.05 / 9=0.006$ ) as level of statistical significance. Based on these results, the change in $\mathrm{TnI}$ for which the risk of mortality was increased, was used as a cut-off value to distinguish a stable TnI 
pattern from TnI patterns with a rise and fall. In assigning each of the patients to either one of the patterns (stable pattern, rise-andfall) patients with only a rise in TnI or only a fall in TnI were taken together with patients with a rise-and-fall pattern; i.e. these patients were considered to actually have a rise-and-fall pattern where the initial rise or the fall was not measured.

\section{Outcomes}

The primary outcome was all-cause mortality within 1 year after surgery. Postoperative myocardial infarction, defined according to the third universal definition, [4] was included as a secondary outcome.

\section{Statistical analysis}

Baseline characteristics were compared between the two different patterns of TnI that we identified. Categorical variables were compared using the $\chi^{2}$ test and age was compared using the two-sample t-test. The number of TnI measurements was compared between these two groups using the Mann-Whitney U test.

The highest TnI value and the absolute and relative change in TnI were then compared between patients who were deceased and alive after 1 year, and between patients with and without postoperative myocardial infarction using the Mann-Whitney U test. We compared 1-year survival between patients with a stable TnI pattern and a rise-and-fall pattern using Kaplan-Meier analysis and the log-rank test.

Cox regression analysis was used to determine the hazard ratio (HZ) for mortality in patients with a rise-and-fall pattern, as compared to a stable TnI pattern. Multivariable cox regression analysis was used to determine the added predictive value of the TnI pattern on 1-year mortality on top of the highest TnI value and RCRI variables. Therefore, we compared the predictive value of a multivariable model including the highest TnI value, RCRI variables and the TnI pattern to a model only including the highest TnI value and RCRI variables using the area under the receiver operating curve (AUROC).

Next, we compared cardiac interventions between the patients with a stable TnI pattern and a rise-and-fall pattern. Furthermore, in the patients with myocardial infarction, we compared 1-year mortality between the patients with a stable TnI pattern and a rise-and-fall pattern using the $\chi^{2}$ test.

Finally, we considered that patients with evident non-cardiac causes of TnI elevation, i.e. patients with sepsis or severe intracranial pathology, may confound the results. Therefore we conducted a sensitivity analysis after exclusion of these patients.

The analysis was performed using SPSS (release 21.0 for Windows). Throughout the analysis a level of significance of 0.05 was used, apart from the aforementioned adaptation of the $\alpha$ to 0.006 in the definition of the TnI pattern.

\section{Results}

During the study period 4105 patients were eligible for inclusion, of which 55 (1\%) patients were excluded: four patients were transferred to another hospital on the day of surgery, 19 patients died on the day of surgery, hence TnI could not be measured in these patients, and 32 patients were lost to follow-up as mortality data could not be obtained from the municipal personal records database. Of the remaining 4050 patients, TnI was measured after surgery in $3224(80 \%)$ patients. TnI was elevated in 715 (22\%) of these 3224 patients. From these 715 patients, 66 were excluded because TnI was only measured once, hence the change in TnI could not be calculated. In addition, in 15 patients the first elevated TnI was measured on clinical indication at $>72 \mathrm{~h}$ after the start of surgery, and was not part of the postoperative care protocol, and therefore they were excluded. In total, 634 patients were included in the analysis (Figure 1).

Figure 2 shows the risk ratios (RR) for 1-year mortality for several cut-off values. The risk of mortality increased significantly with peak TnI values $\geq 90 \mathrm{ng} / \mathrm{L}$ (RR 1.9, 99.4\% CI 1.1-3.1, p=0.001). Furthermore, the risk of mortality increased significantly with an absolute change in TnI of $\geq 200 \mathrm{ng} / \mathrm{L}$ (RR 1.5, 99.4\% CI 1.0-2.2, $\mathrm{p}=0.003$ ), i.e. this was the lowest absolute change at which the risk of mortality was significantly increased. The risk of mortality was not increased for relative changes in TnI. Based on these results, an absolute change in $\mathrm{TnI} \geq 200 \mathrm{ng} / \mathrm{L}$ was used as a cut-off level to distinguish a rise-and-fall pattern from a stable pattern.

Of the 634 patients, 459 patients (72\%) had a stable TnI pattern, 110 patients (17\%) had a rise-and-fall pattern, 26 patients (4\%) had a rising pattern, and 39 patients $(6 \%)$ had a falling pattern. In the further analysis, the patients with a rising pattern or a falling pattern were taken together with the patients with a rise-and-fall pattern. Baseline characteristics, stratified for the different TnI patterns, are given in Table 1. Patients with a rise-and-fall pattern more often had diabetes and renal insufficiency, used more cardiovascular medications, and more often underwent vascular surgery and re-operations as compared to patients

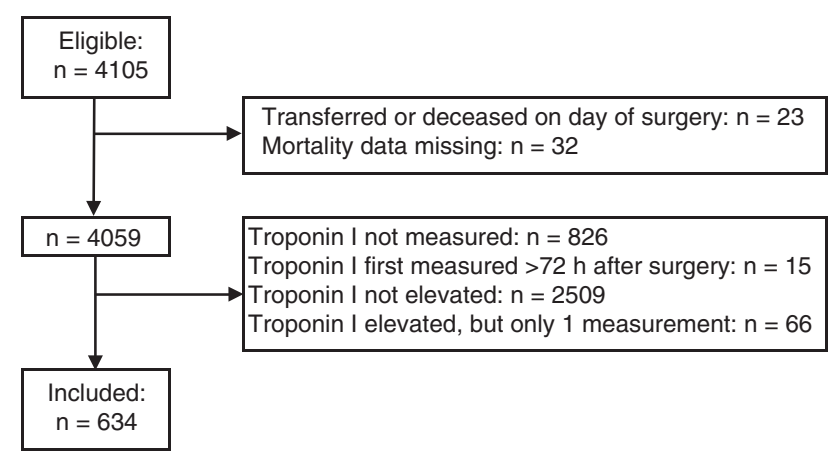

Figure 1: Flow chart of patient inclusion. 


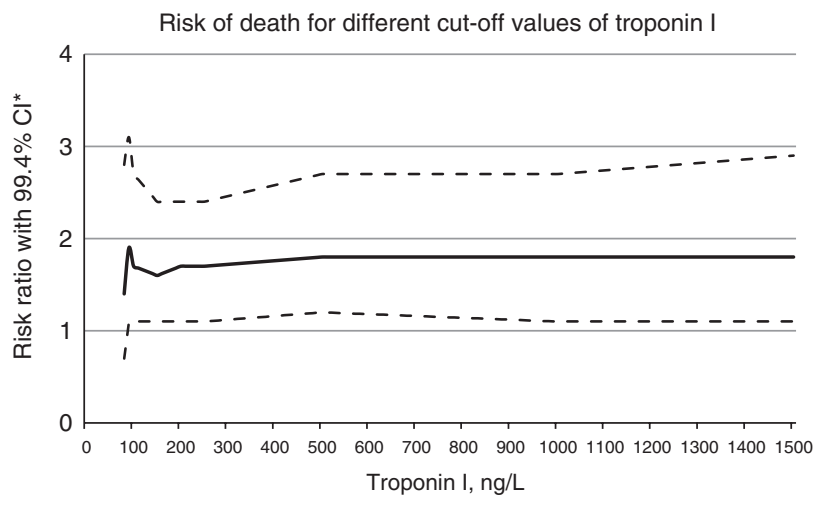

Risk of death for different cut-off values of the absolute change in troponin I

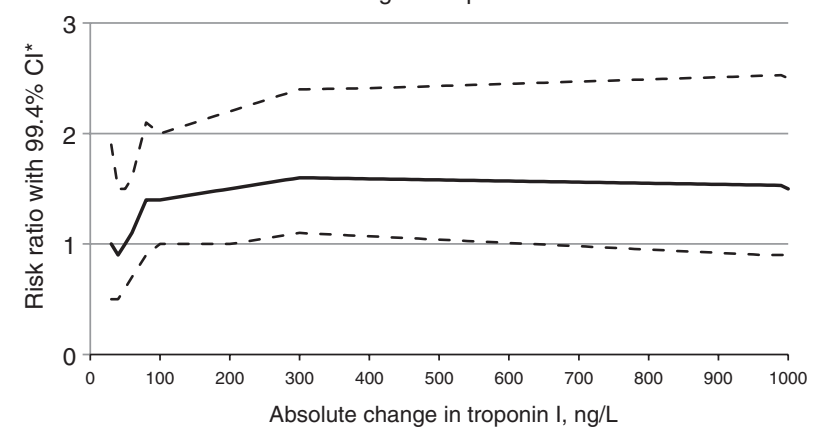

Risk of death for different cut-off values of the relative change in troponin I

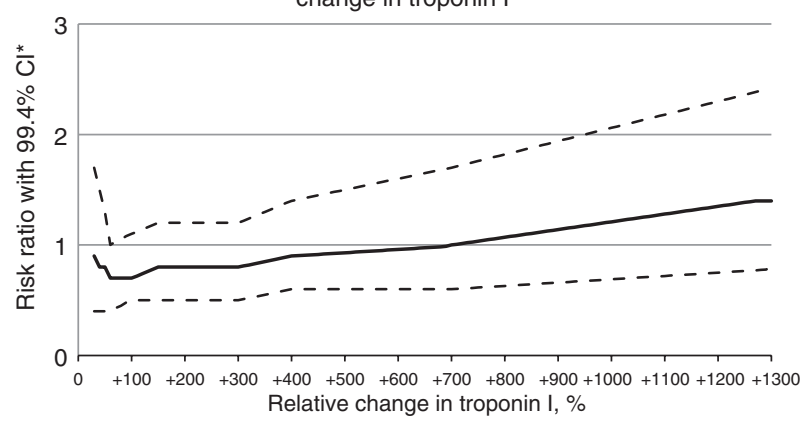

Figure 2: Risk of 1-year mortality for different cut-off values of the highest $\mathrm{Tnl}$ value, the absolute change in Tnl, and the relative change in Tnl.

*Confidence interval.

with a stable $\mathrm{TnI}$ pattern. The median number of $\mathrm{TnI}$ measurements was four [Interquartile range (IQR) 3-5] in patients with a rise-and-fall pattern, as compared to three measurements (IQR 3-3) in patients with a stable pattern.

\section{Primary outcome}

One-year mortality occurred in 155 (24\%) patients. In patients who died, the median highest $\mathrm{TnI}$ value was $190 \mathrm{ng} / \mathrm{L}$ (IQR 100-660 ng/L), as compared to $120 \mathrm{ng} / \mathrm{L}$ in patients who were alive (IQR $80-280 \mathrm{ng} / \mathrm{L}, \mathrm{p}<0.01$ ). The median absolute change in TnI was $110 \mathrm{ng} / \mathrm{L}$ (IQR 50-460 $\mathrm{ng} / \mathrm{L}$ ) in patients who died within 1 year, as compared to $71 \mathrm{ng} / \mathrm{L}$ (IQR $50-180 \mathrm{ng} / \mathrm{L}$ ) in patients who survived $(\mathrm{p}=0.02)$. The median relative change in TnI was $+175 \%$ in patients who died (IQR $+60 \%$ to $+510 \%$ ), as compared to $+243 \%$ in alive patients (IQR $+96 \%$ to $+600 \%)(\mathrm{p}=0.18)$ (Figure 3). One-year survival was better in patients with a stable TnI pattern, as compared to patients with a riseand-fall or falling TnI pattern (Figure 4).

Univariable regression analysis showed that the $\mathrm{HZ}$ for mortality was 1.7 (95\% CI 1.2-2.3, p<0.01) in patients with a rise-and-fall pattern as compared to patients with a stable TnI pattern. After adjustment for the highest TnI value and the variables from the RCRI, the TnI pattern was not associated with mortality (Table 2). Adding the TnI pattern to a model including the highest TnI value and the RCRI variables did not increase the predictive value on mortality: AUROC 0.67 (95\% CI 0.62-0.72) vs. 0.66 (95\% CI 0.61-0.71).

A sensitivity analysis excluding patients with evident non-cardiac causes of $\mathrm{TnI}$ elevation, i.e. intracerebral pathology $(n=6)$ or sepsis $(n=6)$ did not alter the results.

\section{Secondary outcome}

Ninety patients (14\%) fulfilled the criteria of myocardial infarction. In those patients, the median highest TnI value was $745 \mathrm{ng} / \mathrm{L}$ (IQR 160-6335 ng/L), as compared to 120 $\mathrm{ng} / \mathrm{L}$ (IQR 80-260 ng/L) in patients without myocardial infarction $(\mathrm{p}<0.01)$. The median absolute change in TnI was $525 \mathrm{ng} / \mathrm{L}$ (IQR 68-4955 ng/L) in patients with myocardial infarction, as compared to $70 \mathrm{ng} / \mathrm{L}$ (50-160 ng/L) in patients without $(\mathrm{p}<0.01)$. The median relative change in TnI was $+375 \%$ (IQR $+67 \%$ to $+3029 \%$ ) in patients with myocardial infarction, as compared to $+200 \%$ (IQR $+80 \%$ to $+450 \%)$ in patients without $(\mathrm{p}<0.01)$.

Of the 90 patients with postoperative myocardial infarction, 34 patients (38\%) had a stable TnI pattern, and $56(62 \%)$ had a rise-and-fall pattern. In these patients with myocardial infarction, mortality within 1 year occurred in 26 of the 56 patients (46\%) with a rise-and-fall pattern, as compared to nine of the 34 patients $(26 \%)$ with a stable pattern $(p=0.06)$.

\section{Cardiac interventions in patients with different $\mathrm{Tn}$ l patterns}

A cardiac consultation was performed in 130 (74\%) of the 175 patients with a rise-and-fall pattern, as compared 
Table 1: Baseline characteristics, stratified by the pattern of postoperative $\mathrm{Tnl}$ measurements.

\begin{tabular}{|c|c|c|c|}
\hline & $\begin{array}{r}\text { Tn stable: } \\
\Delta \mathrm{Tn}<200 \mathrm{ng} / \mathrm{L} \\
\mathrm{n}=459\end{array}$ & $\begin{array}{r}\text { Tn rise-and-fall: } \\
\begin{array}{r}\Delta \mathrm{Tn} \geq 200 \mathrm{ng} / \mathrm{L} \\
\mathrm{n}=175\end{array}\end{array}$ & p-Value \\
\hline Male & $283(62.0)$ & $98(56.0)$ & 0.19 \\
\hline Mean age, SD & $73.0(8.2)$ & $73.1(8.2)$ & 0.94 \\
\hline Hypertension & $280(61.0)$ & $114(65.1)$ & 0.34 \\
\hline Diabetes & $84(18.3)$ & $46(26.3)$ & 0.03 \\
\hline History of myocardial infarction & $61(13.3)$ & $33(18.9)$ & 0.08 \\
\hline History of coronary revascularization & $84(18.3)$ & $35(20.0)$ & 0.62 \\
\hline Chronic heart failure & $24(5.2)$ & $10(5.7)$ & 0.81 \\
\hline (Paroxysmal) atrial fibrillation & $72(15.7)$ & $23(13.1)$ & 0.42 \\
\hline Pacemaker and/or ICD & $21(4.6)$ & $10(5.7)$ & 0.55 \\
\hline History of cerebrovascular disease & $80(17.4)$ & $39(22.3)$ & 0.16 \\
\hline Chronic obstructive pulmonary disease & $52(11.3)$ & $18(10.3)$ & 0.71 \\
\hline Preoperative renal insufficiency & $85(18.5)$ & $50(28.6)$ & $<0.01$ \\
\hline Peripheral vascular disease & $66(14.4)$ & $34(19.4)$ & 0.12 \\
\hline \multicolumn{4}{|l|}{ Chronic medication use } \\
\hline Beta blockers & $177(38.6)$ & 68 (38.9) & 0.95 \\
\hline Angiotensin renin blockers & $179(39.0)$ & $84(48.0)$ & 0.04 \\
\hline Calcium channel blockers & $89(19.4)$ & $36(20.6)$ & 0.74 \\
\hline Diuretics & $148(32.2)$ & $57(32.6)$ & 0.94 \\
\hline Statins & $187(40.7)$ & $89(50.9)$ & 0.02 \\
\hline Aspirin & $159(34.6)$ & $83(47.4)$ & 0.03 \\
\hline Warfarins & $70(15.3)$ & $18(10.3)$ & 0.11 \\
\hline \multicolumn{4}{|l|}{ ASA class $\Delta$} \\
\hline 1 & $36(7.8)$ & $9(5.1)$ & 0.51 \\
\hline 2 & $265(57.7)$ & $98(56.0)$ & \\
\hline$\geq 3$ & $158(34.4)$ & $68(38.9)$ & \\
\hline General anesthesia & $442(96.3)$ & $170(97.1)$ & 0.60 \\
\hline High-risk surgery & $200(43.6)$ & $83(47.4)$ & 0.38 \\
\hline Emergency surgery & $148(32.2)$ & $65(37.1)$ & 0.24 \\
\hline Re-operation within 1 year & $99(21.6)$ & $55(31.4)$ & 0.01 \\
\hline \multicolumn{4}{|l|}{ Surgical specialty } \\
\hline General & $162(35.3)$ & $57(32.6)$ & 0.16 \\
\hline Neuro & $89(19.4)$ & $28(16.0)$ & \\
\hline Vascular & $81(17.6)$ & $49(28.0)$ & \\
\hline ENT and dental & $45(9.8)$ & $13(7.4)$ & \\
\hline Orthopedic & $54(12.0)$ & $20(11.4)$ & \\
\hline Gynecology/urologic & $28(6.1)$ & $8(4.6)$ & \\
\hline
\end{tabular}

Figures are numbers of patients (\%), unless indicated otherwise. SD, standard deviation; ICD, implantable cardiovertor defibrillator; $\Delta$, Classification System by the American Society of Anesthesiologists; ENT, ear nose throat.

to $141(31 \%)$ of the 459 patients with a stable pattern $(\mathrm{p}<0.01)$. This was followed by an intervention in 61 patients $(35 \%)$ as compared to 42 patients $(9 \%)(p<0.01)$. New medication or a dose increase, including beta-blockers, renin-angiotensin inhibitors, calcium channel blockers, diuretics, statins, aspirin, other antiplatelet drugs, and (low molecular weight) heparin, was prescribed in 60 patients with a rise-and-fall pattern (34\%), as compared to 37 patients $(8 \%)$ with a stable pattern $(p<0.01)$. Of the patients with a rise-and-fall pattern, more patients were transferred to the coronary care unit or medium care unit for cardiac monitoring, more patients underwent coronary angiography, and more patients underwent percutaneous coronary intervention (Table 3).

\section{Discussion}

To help clinicians in interpreting postoperative troponin elevation, we described the kinetic changes of TnI in patients with myocardial injury after noncardiac surgery using troponin values that were routinely measured as part of a clinical protocol. In addition, the added predictive 


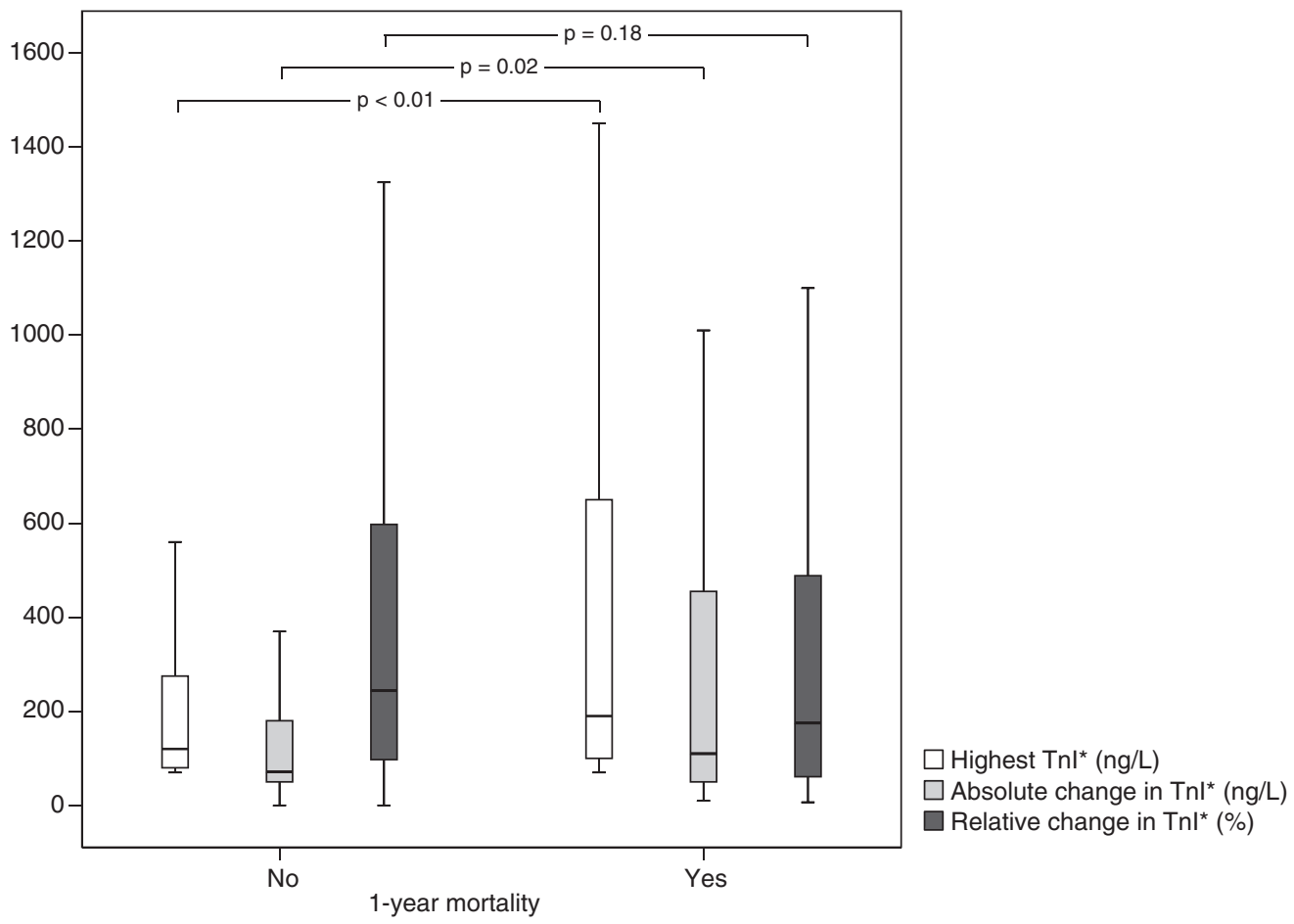

Figure 3: The median highest troponin I value, the median absolute change in troponin I, and the median relative change in troponin I in patients who died within 1 year and patients who survived.

*Troponin I.

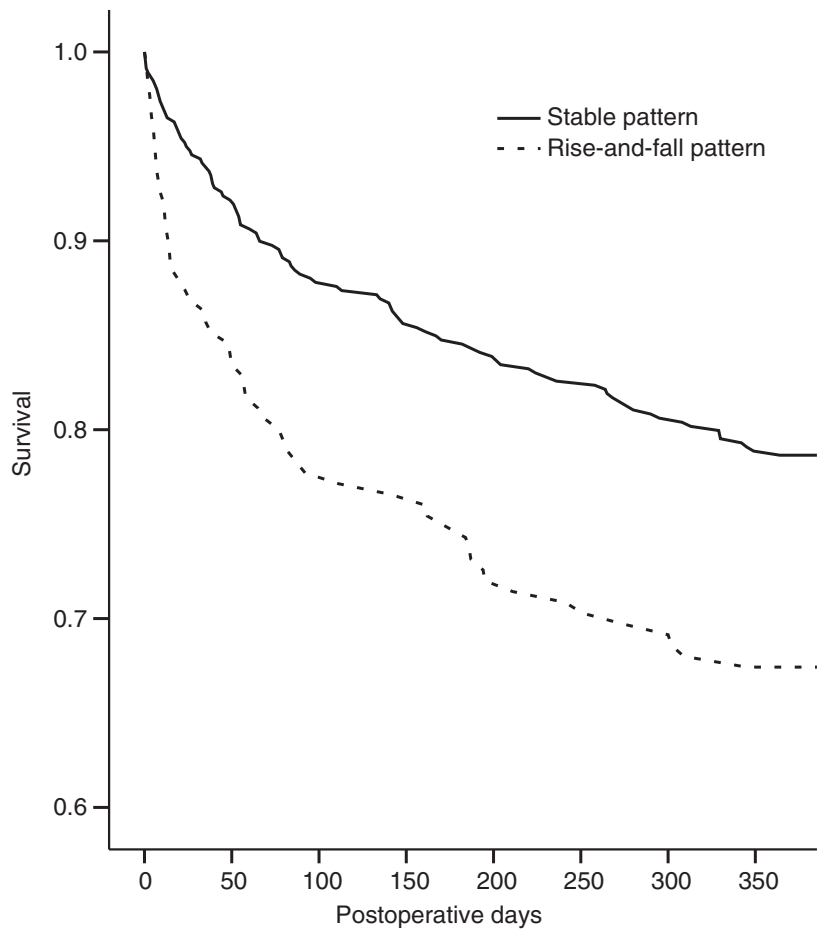

Figure 4: Survival curve of patients with different Tnl patterns. A stable troponin I pattern was defined as an absolute change in TnI $<200 \mathrm{ng} / \mathrm{L}$, and a rise-and-fall pattern was defined as an absolute change in $\mathrm{Tnl} \geq 200 \mathrm{ng} / \mathrm{L}$. value of kinetic changes in TnI after noncardiac surgery to predict 1-year mortality was determined.

Among patients with myocardial injury after surgery, we found that an absolute change in TnI of at least $200 \mathrm{ng} / \mathrm{L}$ was associated with mortality. Such a change showing a rise-and-fall pattern was found in $28 \%$ of patients. Adding the absolute TnI change to a model including the highest TnI level, did not improve the ability to predict mortality. Relative changes in TnI did not predict mortality.

\section{Limitations}

This study has some obvious limitations. First of all, since TnI was not measured before surgery, the results of this study cannot be used to interpret postoperative TnI values in relation to preoperative values. The patients with a stable TnI pattern after surgery may in fact have had a rise-and-fall pattern in relation to a preoperative value, i.e. in those patients the perioperative rise and fall may have been missed because TnI was not measured before surgery. However, this study determined the additional value of TnI kinetics in patients in whom troponin is only measured after surgery, like in our standard postoperative care. In the future, it would be of interest to study 
Table 2: The hazard ratio of 1-year mortality in patients with a riseand-fall pattern as compared to a stable TnI pattern, adjusted for the highest Tnl value and variables from the RCRI.

\begin{tabular}{lrrr}
\hline & \multicolumn{3}{l}{ Adjusted analysis } \\
\cline { 2 - 4 } & HR & $95 \% \mathrm{Cl}$ & $\mathrm{p}-$ Value \\
\hline Tnl pattern & & & \\
Stable (absolute change in TnI $<200 \mathrm{ng} / \mathrm{L})$ & Ref & & \\
Rise-and-fall (absolute change in & 0.5 & $0.3-1.1$ & 0.11 \\
Tnl $\geq 200 \mathrm{ng} / \mathrm{L})$ & & & \\
Highest Tnl value, ng/L & & & \\
$70-79$ & Ref & & \\
$80-89$ & 0.7 & $0.3-1.5$ & 0.35 \\
$90-99$ & 1.5 & $0.7-3.5$ & 0.31 \\
$100-129$ & 1.4 & $0.7-2.7$ & 0.31 \\
$130-169$ & 1.0 & $0.5-2.1$ & 0.96 \\
$170-259$ & 1.9 & $1.0-3.7$ & 0.06 \\
$260-499$ & 1.9 & $0.9-4.3$ & 0.10 \\
$500-1499$ & 4.1 & $1.5-10.7$ & $<0.01$ \\
$\geq 1500$ & 4.3 & $1.7-11.0$ & $<0.01$ \\
RCRI & & & \\
High-risk surgery & 1.0 & $0.7-1.4$ & 0.89 \\
Ischemic heart disease & 0.9 & $0.6-1.3$ & 0.57 \\
Chronic heart failure & 1.1 & $0.6-2.2$ & 0.70 \\
Cerebrovascular disease & 1.1 & $0.6-1.7$ & 0.84 \\
Preoperative renal insufficiency & 2.1 & $1.5-3.0$ & $<0.01$ \\
Insulin use & 1.0 & $0.6-1.7$ & 0.98 \\
\hline
\end{tabular}

$\mathrm{HR}$, hazard ratio; $\mathrm{Cl}$, confidence interval.

postoperative troponin changes in relation to preoperative values. Second, because follow-up of elevated TnI levels was not strictly protocolized, yet was performed at discretion of the attending cardiologist, $\mathrm{TnI}$ was not measured every 4-6 $\mathrm{h}$ in all patients. Therefore, a rise-and-fall pattern may have been missed in some patients. Finally, the analysis was not adjusted for treatment interventions that were undertaken in patients with myocardial injury and that may have influenced prognosis. This may have led to an underestimation of the predictive value of $\mathrm{TnI}$ kinetics.

\section{Literature}

Kinetics of troponin have been well studied and implemented in the diagnosis of myocardial infarction in patients suspected of acute coronary syndrome in a non-perioperative setting. Although a relative change in troponin of $>20 \%$ is considered to be diagnostic for myocardial infarction in patients after percutaneous coronary intervention or reinfarction, [4] absolute troponin changes are superior to relative changes in discriminating myocardial infarction in patients presenting in the emergency department [10, 12-15]. Absolute TnI changes discriminate well between acute myocardial infarction and cardiac noncoronary artery disease, and are useful in selecting patients who require invasive treatment [10]. To predict outcome however, TnI changes do not improve risk prediction as compared to absolute troponin values and clinical risk factors [16].

Few studies have evaluated troponin kinetics in the perioperative period. Kavsak and colleagues studied relative changes of postoperative troponin $\mathrm{T}(\mathrm{TnT})$ in vascular surgery patients by using two cut-off levels that were

Table 3: Cardiology consultation and cardiac interventions, stratified by the pattern of postoperative Tnl measurements.

\begin{tabular}{|c|c|c|c|}
\hline & $\begin{array}{r}\text { Tn stable: } \\
\Delta \operatorname{Tn}<200 \mathrm{ng} / \mathrm{L} \\
\mathrm{n}=459\end{array}$ & $\begin{array}{r}\text { Tn rise-and-fall: } \\
\begin{array}{r}\Delta \mathrm{Tn} \geq 200 \mathrm{ng} / \mathrm{L} \\
\mathrm{n}=\mathbf{1 7 5}\end{array}\end{array}$ & p-Value \\
\hline Cardiology consultation & $141(30.7)$ & $130(74.3)$ & $<0.01$ \\
\hline Any intervention & $42(9.2)$ & $61(34.9)$ & $<0.01$ \\
\hline New prescribed medication or dose increase & $37(8.1)$ & $60(34.3)$ & $<0.01$ \\
\hline Beta blockers & $12(2.6)$ & $37(21.1)$ & $<0.01$ \\
\hline Other antihypertensive drugs ${ }^{a}$ & $7(1.5)$ & $12(6.9)$ & $<0.01$ \\
\hline Statins & $5(1.1)$ & $16(9.1)$ & $<0.01$ \\
\hline Aspirin & $10(2.3)$ & $24(13.7)$ & $<0.01$ \\
\hline Other antiplatelet drugs & $0(0)$ & $14(8.0)$ & $<0.01$ \\
\hline (Low molecular weight) heparin & $10(2.3)$ & $15(8.6)$ & $<0.01$ \\
\hline Other & $13(2.8)$ & $10(5.7)$ & 0.08 \\
\hline Cardiac monitoring & $4(0.9)$ & $11(6.3)$ & $<0.01$ \\
\hline Coronary angiography & $2(0.4)$ & $12(6.9)$ & $<0.01$ \\
\hline Percutaneous coronary intervention & $1(0.2)$ & $7(4.0)$ & $<0.01$ \\
\hline Coronary artery bypass grafting & $0(0)$ & $1(0.6)$ & 0.11 \\
\hline
\end{tabular}

Figures are numbers of patients (\%). ancluding renin angiotensin blockers, calcium channel blockers and diuretics. 
based on previous studies in healthy volunteers and patients suspected of acute coronary syndrome [17]. They reported that half of the patients with postoperative troponin elevation had a relative change in $\mathrm{TnT}>85 \%$ as compared to the preoperative TnT level, and one in four patients had a relative TnT change $>242 \%$. Gillmann and colleagues studied both absolute and relative changes in patients undergoing vascular surgery, and found that an absolute change in TnT of as small as $6 \mathrm{ng} / \mathrm{L}$, as compared to the preoperative TnT level, was independently associated with major adverse cardiac events, and that adding the $\mathrm{TnT}$ absolute change to clinical risk factors improved the predictive accuracy for major adverse cardiac events [18]. These results differ from our study, which may be explained by the fact that they calculated postoperative troponin changes in relation to preoperative troponin levels, while we only studied postoperative troponin values. Furthermore, Gillmann and colleagues reported that absolute $\mathrm{TnT}$ changes were superior to relative $\mathrm{TnT}$ changes for perioperative risk prediction, which is in concordance with our finding that relative changes were not related to mortality [18].

\section{Clinical implications}

Several studies have shown that myocardial injury after noncardiac surgery as measured by troponin elevation, is an independent predictor of mortality. Yet, because troponin elevation may occur in a variety of conditions and the cause of myocardial injury is often unclear, it is not known in which patients prognosis can be improved. Therefore, improved risk stratification of patients with myocardial injury is warranted. Since kinetic changes of cardiac biomarkers, described as a typical rise and fall, are the cornerstone in the diagnosis of myocardial infarction in the non-perioperative setting, kinetic changes of troponin may be considered important in the further assessment of prognosis in patients with myocardial injury after surgery. Although we found that postoperative absolute TnI changes of more than $200 \mathrm{ng} / \mathrm{L}$ are associated with mortality, these absolute TnI changes had no added value to predict mortality in addition to the highest TnI level. Since absolute changes are larger in patients with high TnI levels, a large absolute change in TnI is inherent to high TnI values, which may explain why TnI changes alone do not have any added predictive value to absolute values. Furthermore, relative changes were not associated with mortality, which may be explained by the fact that relative changes cannot distinguish a patient with low TnI levels, and therefore a relatively good prognosis, from a patient with very high $\mathrm{TnI}$ levels and a poor prognosis, e.g. the relative change in $\mathrm{TnI}$ from $80 \mathrm{ng} / \mathrm{L}$ to $120 \mathrm{ng} / \mathrm{L}$ is similar to the relative change in $\mathrm{TnI}$ from $2000 \mathrm{ng} / \mathrm{L}$ to 3000 (both $+50 \%$ ).

Of note, $38 \%$ of the patients with a postoperative myocardial infarction did not show a rise-and-fall in TnI as determined in this study (absolute change $>200 \mathrm{ng} / \mathrm{L}$ ), although a rise-and-fall is required according to the third universal definition of myocardial infarction. This finding highlights an important problem in interpreting the universal definition: since the rise-and-fall that is required to diagnose myocardial infarction is not defined, in fact any change in troponin may be considered a rise-andfall, hence even patients with very small changes may be diagnosed with myocardial infarction. This issue may be further complicated by the use of high sensitivity troponin assays: it has been shown that when high sensitivity troponin assays are used, the use of troponin changes on top of absolute troponin values improves specificity but reduces sensitivity in patients who are suspected of myocardial infarction in the emergency department [19]. In the perioperative setting, the use of troponin changes as detected by high sensitivity assays are subject to further investigation.

Although only $43 \%$ of all patients had cardiology consultation, and interventions were only performed in $24 \%$ of patients, this was done more often in patients with a rise-and-fall pattern (in $74 \%$ and $35 \%$ of patients, respectively). Apparently, cardiologists were more likely to adjudicate a TnI rise-and-fall pattern as a myocardial infarction.

\section{Conclusions}

Among patients with myocardial injury after noncardiac surgery, as detected by routine postoperative TnI measurements, kinetics of postoperative TnI showed a rise-andfall pattern in one third of patients. An absolute change in TnI of more than $200 \mathrm{ng} / \mathrm{L}$ was associated with 1-year mortality, but had no added predictive value in addition to the highest $\mathrm{TnI}$ level. Therefore, we consider TnI kinetics not useful for further stratification of mortality risk in patients with postoperative myocardial injury.

Acknowledgments: We acknowledge Wietze Pasma and the trial office nurses under the direction of Sandra Numan for their contributions in data collection.

Author contributions: All the authors have accepted responsibility for the entire content of this submitted manuscript and approved submission. 
Research funding: This study was funded by a grant from the International Anesthesia Research Society (Clinical Scholar Research Award 2011 to dr. Van Klei), by a grant from the Friends of the University Medical Center Utrecht foundation/the Dirkzwager-Assink Fund to dr. Van Klei and by departmental sources.

Employment or leadership: None declared.

Honorarium: None declared.

Competing interests: The funding organization(s) played no role in the study design; in the collection, analysis, and interpretation of data; in the writing of the report; or in the decision to submit the report for publication.

\section{References}

1. Levy MM, Heels-Ansdell D, Hiralal R, Bhandari M, Guyatt G, Yusuf $S$, et al. Prognostic value of troponin and creatine kinase muscle and brain isoenzyme measurement after noncardiac surgery. Anesthesiology 2011;114:796-806.

2. The Vascular Events in Noncardiac Surgery Patients Cohort Evaluation (VISION) Study Investigators. Association between postoperative troponin levels and 30-day mortality among patients undergoing noncardiac surgery. J Am Med Assoc 2012;307:2295-304.

3. van Waes JA, Nathoe HM, de Graaff JC, Kemperman H, de Borst GJ, Peelen LM, et al. Myocardial injury after noncardiac surgery and its association with short-term mortality. Circulation 2013;127:2264-71.

4. Thygesen K, Alpert JS, Jaffe AS, Simoons ML, Chaitman BR, White $H D$, et al. Third universal definition of myocardial infarction. Circulation 2012;126:2020-35.

5. Kristensen SD, Knuuti J, Saraste A, Anker S, Botker HE, Hert S de, et al. 2014 ESC/ESA Guidelines on non-cardiac surgery: cardiovascular assessment and management. The joint task force on non-cardiac surgery: cardiovascular assessment and management of the European Society of Cardiology (ESC) and the European Society of Anaesth. Eur J Anaesthesiol 2014;31:517-73.

6. Longo DL, Devereaux PJ, Sessler DI. Cardiac complications in patients undergoing major noncardiac surgery. N Engl J Med 2015;373:2258-69.

7. Fleisher LA, Fleischmann KE, Auerbach AD, Barnason SA, Beckman JA, Bozkurt B, et al. 2014 ACC/AHA guideline on perioperative cardiovascular evaluation and management of patients undergoing noncardiac surgery: a report of the American College of Cardiology/American Heart Association task force on practice guidelines. Circulation 2014;130:2246-64.
8. van Waes JAR, Grobben RB, Nathoe HM, Kemperman H, de Borst GJ, Peelen LM, et al. One-year mortality, causes of death, and cardiac interventions in patients with postoperative myocardial injury. Anesth Analg 2016;123:29-37.

9. Hamm CW. Cardiac troponin elevations in patients without acute coronary syndrome. Circulation 2002;106:2871-2.

10. Haaf P, Drexler B, Reichlin T, Twerenbold R, Reiter M, Meissner J, et al. High-sensitivity cardiac troponin in the distinction of acute myocardial infarction from acute cardiac noncoronary artery disease. Circulation 2012;126:31-40.

11. Lee TH, Marcantonio ER, Mangione CM, Thomas EJ, Polanczyk $\mathrm{CA}$, Cook EF, et al. Derivation and prospective validation of a simple index for prediction of cardiac risk of major noncardiac surgery. Circulation 1999;100:1043-9.

12. Biener M, Mueller M, Vafaie M, Jaffe AS, Widera C, Katus HA, et al. Diagnostic performance of rising, falling, or rising and falling kinetic changes of high-sensitivity cardiac troponin $\mathrm{T}$ in an unselected emergency department population. Eur Heart Journal Acute Cardiovasc Care 2013;2:314-22.

13. Irfan A, Reichlin T, Twerenbold R, Meister M, Moehring B, Wildi $\mathrm{K}$, et al. Early diagnosis of myocardial infarction using absolute and relative changes in cardiac troponin concentrations. Am J Med 2013;126:781-8.

14. Mueller M, Biener M, Vafaie M, Doerr S, Keller T, Blankenberg S, et al. Absolute and relative kinetic changes of high-sensitivity cardiac troponin $\mathrm{T}$ in acute coronary syndrome and in patients with increased troponin in the absence of acute coronary syndrome. Clin Chem 2012;58:209-18.

15. Reichlin T, Irfan A, Twerenbold R, Reiter M, Hochholzer W, Burkhalter $\mathrm{H}$, et al. Utility of absolute and relative changes in cardiac troponin concentrations in the early diagnosis of acute myocardial infarction. Circulation 2011;124:136-45.

16. Biener M, Mueller M, Vafaie M, Jaffe AS, Katus HA, Giannitsis E. Prognostic performance of high-sensitivity cardiac troponin $T$ kinetic changes adjusted for elevated admission values and the GRACE score in an unselected emergency department population. Clin Chim Acta 2014;435:29-35.

17. Kavsak PA, Walsh M, Srinathan S, Thorlacius L, Lurati Buse G, Botto F, et al. High sensitivity troponin T concentrations in patients undergoing noncardiac surgery: a prospective cohort study. Clin Biochem 2011;44:1021-4.

18. Gillmann H-J, Meinders A, Grohennig A, Larmann J, Bünte C, Calmer S, et al. Perioperative levels and changes of high-sensitivity troponin $\mathrm{T}$ are associated with cardiovascular events in vascular surgery patients. Crit Care Med 2014;42:1498-506.

19. Aldous SJ, Florkowski CM, Crozier IG, Elliott J, George P, Lainchbury JG, et al. Comparison of high sensitivity and contemporary troponin assays for the early detection of acute myocardial infarction in the emergency department. Ann Clin Biochem 2011;48:241-8. 